ARTIGO ORIGINAL

ORIGINAL ARTICLE

\title{
Modelo de impacto orçamentário do trastuzumabe subcutâneo comparado com o intravenoso no tratamento de câncer de mama HER-2 positivo no Sistema de Saúde Suplementar brasileiro
}

\author{
Budget impact model of subcutaneous compared with \\ intravenous trastuzumab in the treatment of HER-2 positive \\ breast cancer in the Brazilian Private Healthcare System \\ Diego Kashiura', Paulo Vitor dos Santos Souza², Ligia Fernande Yoshida',

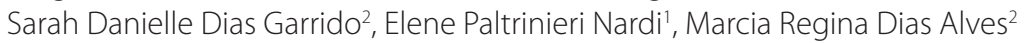

DOI: 10.21115/JBES.v10.n3.p269-77

\section{Palavras-chave:}

trastuzumabe, subcutâneo, intravenoso, custos, Brasil, Sistema de Saúde Suplementar

\section{Keywords:}

trastuzumab, subcutaneous, intravenous, costs, Brazil, Private Healthcare System

\section{RESUMO}

Objetivo: Estimar o impacto orçamentário do trastuzumabe subcutâneo, comparado com o intravenoso, no Sistema de Saúde Suplementar (SSS) para o tratamento do câncer de mama inicial e metastático HER-2 positivo. Métodos: Foi realizada uma análise de impacto orçamentário na perspectiva do SSS. Os tratamentos de câncer de mama inicial foram trastuzumabe em monoterapia e trastuzumabe com paclitaxel ou docetaxel. No câncer de mama metastático, considerou-se trastuzumabe associado com docetaxel ou paclitaxel na primeira linha e em monoterapia na segunda linha. Para ambos, comparou-se a substituição do trastuzumabe intravenoso pelo subcutâneo. Uma pesquisa com 28 operadoras mapeou taxas, preços e tabelas de referência usadas para o reembolso de medicamentos, materiais e procedimentos. Uma equipe multidisciplinar estimou o uso de recursos. Custos médicos diretos foram incluídos e os preços foram obtidos de fontes públicas. O horizonte temporal foi cinco anos e considerou-se a incorporação progressiva de trastuzumabe subcutâneo com 20\% no primeiro ano até atingir 100\%. Resultados: Estimaram-se 31.589 pacientes com câncer de mama no SSS em cinco anos. O uso progressivo de trastuzumabe subcutâneo em comparação com o intravenoso levou a uma economia de 962,7 mBRL, 14,5 mBRL, 1,5 mBRL e 0,2 mBRL no SSS, para operadoras de saúde de grande, médio e pequeno porte, respectivamente. Materiais e procedimentos de suporte com taxas médicas foram os parâmetros mais sensíveis. Conclusão: 0 trastuzumabe subcutâneo em comparação com o intravenoso pode levar a uma economia de até 962,7 mBRL no tratamento do câncer de mama no SSS.

\begin{abstract}
Objective: To estimate the budgetary impact of subcutaneous trastuzumab, compared with intravenous trastuzumab, in the Brazilian Private Healthcare System (PHS), to treat early and metastatic HER-2 positive breast cancer. Methods: Budgetary impact analysis was performed in PHS perspective. For early breast cancer, treatment options were trastuzumab monotherapy, and trastuzumab plus paclitaxel or docetaxel, after adjuvant chemotherapy. For metastatic breast cancer, trastuzumab was combined with docetaxel or paclitaxel in the first line, and it was given in monotherapy in the second line. For both, a comparison of switching from intravenous to subcutaneous therapy was made. A survey was performed with 28 health maintenance organizations to map fees and sources used for reimbursement of drugs, materials and procedures. Direct medical costs were included.
\end{abstract}

Recebido em: 31/10/2018. Aprovado para publicação em: 02/01/2019

1. Evidências - Kantar Health São Paulo, SP, Brasil.

2. F. Hoffmann-La Roche Ltd, São Paulo, SP, Brasil.

Auxílio: Este estudo foi realizado pela Evidências - Kantar Health, com suporte financeiro da F. Hoffmann-La Roche.

Conflitos de interesse: Todos os autores são funcionários da F. Hoffmann-La Roche ou da Evidências - Kantar Health.

Congressos: Apresentado na ISPOR Europe 2018.

tor correspondente: Diego Kashiura. Avenida Engenheiro Billings, 1729, Jaguaré, São Paulo, SP. CEP: 05321-900.

Telefax: +55 (11) 3719-4790. E-mail: diego.kashiura@roche.com 


\begin{abstract}
Time horizon was 5 years and market share of subcutaneous trastuzumab vary from 20\% to 100\%. Results were presented according to size of health maintenance organizations and a deterministic sensitivity analysis was conducted to analyze model robustness. Results: In 5 years, 31,589 breast cancer patients were estimated for the PHS, 284 patients for big, 30 patients for medium, and 6 patients for small HMOs. The progressive uptake of subcutaneous trastuzumab, compared with intravenous formulation, can save up to $962,7 \mathrm{mBRL}, 14,5 \mathrm{mBRL}, 1,5 \mathrm{mBRL}$ and 0,2 mBRL, respectively. Materials and support procedures along with medical fees were the most sensitive parameters. Conclusion: Compared with the intravenous administration, subcutaneous trastuzumab can save up to 962,7 mBRL costs in the breast cancer treatment in the Brazilian PHS.
\end{abstract}

\section{Introdução}

O câncer de mama é o câncer mais comum entre as mulheres no Brasil e no mundo, excetuando-se o câncer de pele não melanoma. Estima-se que, em 2012, 1,67 milhão de muIheres foram diagnosticadas com câncer de mama e, dessas, 521.907 foram a óbito devido à doença (WHO, 2012). No Brasil, o Instituto Nacional do Câncer José Alencar Gomes da Silva (Inca) estimou 59.700 novos casos em 2018 (Inca, 2018).

Aproximadamente $20 \%$ dessas mulheres possuem o status HER-2 positivo, característica de tumores que está associada a maior agressividade da doença, maior taxa de recorrência e maior índice de mortalidade (Arias et al., 2017, Mitri et al., 2012). O trastuzumabe é um anticorpo monoclonal humanizado recombinante, desenvolvido por engenharia genética e que se liga seletivamente ao domínio extracelular da proteína do receptor-2 do fator de crescimento epidérmico humano (HER-2) (Hudis et al., 2007). Ele se mostrou eficaz no tratamento de pacientes tanto com câncer de mama inicial (eBC - early breast cancer) quanto com câncer metastático ( $m B C$ - metastatic breast cancer) HER-2 positivos (Cobleigh et al., 1999; Jackisch et al., 2016; Perez et al., 2014; Slamon et al., 2001; Cameron et al., 2017).

Para a doença inicial, ele é indicado para o tratamento combinado com quimioterapia e também em monoterapia após adjuvância. Em doença metastática, ele possui indicação para primeira linha em combinação com quimioterapia e em segunda linha como agente único (F. Hoffmann-La Roche Ltd, 2018a). Ao tratamento com trastuzumabe, tanto na doença inicial quanto na primeira linha da doença metastática, pode-se combinar o pertuzumabe ao esquema de tratamento para promover o duplo bloqueio de HER-2 (F. Hoffmann-La Roche Ltd, 2018b).

Atualmente, o trastuzumabe possui apresentações para administrações intravenosa (IV) e subcutânea (SC) (F. Hoffmann-La Roche Ltd, 2018a). O estudo HANNAH, de fase III, aberto, randomizado e multicêntrico, demonstrou que trastuzumabe SC tem perfil farmacocinético e eficácia não inferior ao trastuzumabe IV com um perfil de segurança similar (Ismael et al., 2012).

A administração SC deve ser feita com dose fixa de 600 mg a cada três semanas sem necessidade de dose de ataque, ao contrário de trastuzumabe IV com dose de ataque de $8 \mathrm{mg} / \mathrm{kg}$ com base no peso do paciente, seguida por doses de manutenção de $6 \mathrm{mg} / \mathrm{kg}$ a cada três semanas ( $\mathrm{F}$. Hoffmann-La Roche Ltd, 2018a).

A substituição de trastuzumabe IV pela administração SC potencialmente reduz o tempo de preparo da aplicação, reduz o risco de erro de medicação, diminui o tempo gasto pelos profissionais de saúde para infusão da droga e otimiza o uso dos recursos médicos. Esses fatores combinados podem resultar em redução dos custos de administração se comparada à administração IV (Tjalma el al., 2018). Além disso, o estudo PREFHER demonstrou que a forma SC é a preferida pelos pacientes por causar menos desconforto e dor, e pela economia de tempo. Portanto, a terapia SC pode impactar positivamente todos stakeholders envolvidos no sistema de saúde: pacientes, profissionais de saúde, hospitais e outros (Tjalma et al., 2018).

Considerando a possível redução de custo ao utilizar trastuzumabe SC em substituição do IV, o objetivo deste estudo é realizar uma análise de impacto orçamentário do tratamento de trastuzumabe SC em comparação com trastuzumabe IV no tratamento do câncer de mama inicial e metastático HER-2 positivo sob a perspectiva do Sistema de Saúde Suplementar brasileiro.

\section{Métodos}

Este modelo de impacto orçamentário avaliou o impacto da incorporação de trastuzumabe SC no Sistema de Saúde Suplementar brasileiro de forma progressiva, comparado à sua apresentação IV, para o tratamento de pacientes com câncer de mama inicial ou metastático HER-2 positivo. O desenvolvimento deste modelo seguiu as orientações das diretrizes da International Society for Pharmacoeconomics and Outcomes Research (ISPOR) e do Ministério da Saúde (Sullivan et al., 2014; Brasil, 2014).

\section{População elegível}

A estimativa da população elegível ao tratamento com trastuzumabe IV ou SC foi realizada a partir do método epidemiológico, no qual os dados de incidência e/ou prevalência da condição estudada são aplicados nos dados populacionais das perspectivas de interesse (Brasil, 2014). 
A estimativa do número de beneficiários do Sistema de Saúde Suplementar para os anos de 2018 a 2022 foi feita a partir dos dados publicados pela Agência Nacional de Saúde Suplementar (ANS). Em 2017, o número total de beneficiários foi de 25.337.790 mulheres (ANS, 2017b). Uma vez que a taxa de crescimento variou de -3,2\% (dezembro/2016) até 0,1\% (junho/2018), adotou-se uma taxa de crescimento de 0,9\% do número de beneficiários que acompanha a estimativa de crescimento populacional divulgada pelo Instituto Brasileiro de Geografia Estatística (IBGE).

Para cada porte de operadora de saúde, estimou-se o número médio de beneficiários a partir dos dados de todas as operadoras de saúde que se enquadram em cada categoria, disponíveis no TabNet da ANS (ANS, 2017a). Para determinar a quantidades de mulheres, utilizou-se a distribuição do Sistema de Saúde Suplementar completo (53\% de mulheres). Segundo o Inca, a incidência de câncer de mama no Brasil é de 56,33 casos a cada 100 mil mulheres (Inca, 2018). Entre essas pacientes, estima-se que 20\% sejam HER-2 positivas (Arias et al., 2017). Quanto ao diagnóstico, 96\% das pacientes são diagnosticadas em estágio I-III e 4\% em estágio IV (Liedke et al., 2014).

Para estimar o número de pacientes com câncer de mama inicial, considerou-se a sobrevida de pacientes diagnosticados em até um ano publicada por um estudo retrospectivo brasileiro que mostrou que $100 \%$ das pacientes diagnosticados em até um ano continuam vivas (Liedke et al., 2014).

Considerou-se, ainda, a incidência de câncer de mama HER-2 positivo em estágio inicial da doença na Saúde Suplementar. O número total de pacientes com câncer de mama inicial elegíveis ao tratamento com trastuzumabe foi estimado a partir da soma entre os pacientes diagnosticados em cada ano e dos pacientes diagnosticados em até seis anos antes do ano de análise que não apresentaram progressão da doença (Figura 1), conforme curva de SLD apresentada pela coorte retrospectiva de Liedke et al. (2014), que analisou prontuários de 2001 a 2006, permitindo avaliação de dados de até seis anos de histórico.

Para a estimativa do número de pacientes com câncer de mama metastático elegíveis ao tratamento com trastuzumabe em primeira linha, considerou-se a quantidade de pacientes diagnosticados com doença metastática a cada ano e a quantidade de pacientes com doença inicial, que evoluíram para doença metastática.

Foram utilizadas as proporções de pacientes que progrediram para doença metastática de 1\%,7\%, 3\%, 2\%, 6\% e 1\% para um, dois, três, quatro, cinco e seis anos entre o diagnóstico e o início da doença, respectivamente. Tais proporções foram estimadas a partir da curva de SLD de Perez et al. (2014). Em segunda linha de tratamento, a estimativa foi feita a partir do número de pacientes que estavam recebendo primeira linha e apresentaram progressão da doença. As proporções de pacientes que progrediram para segunda linha de trata-

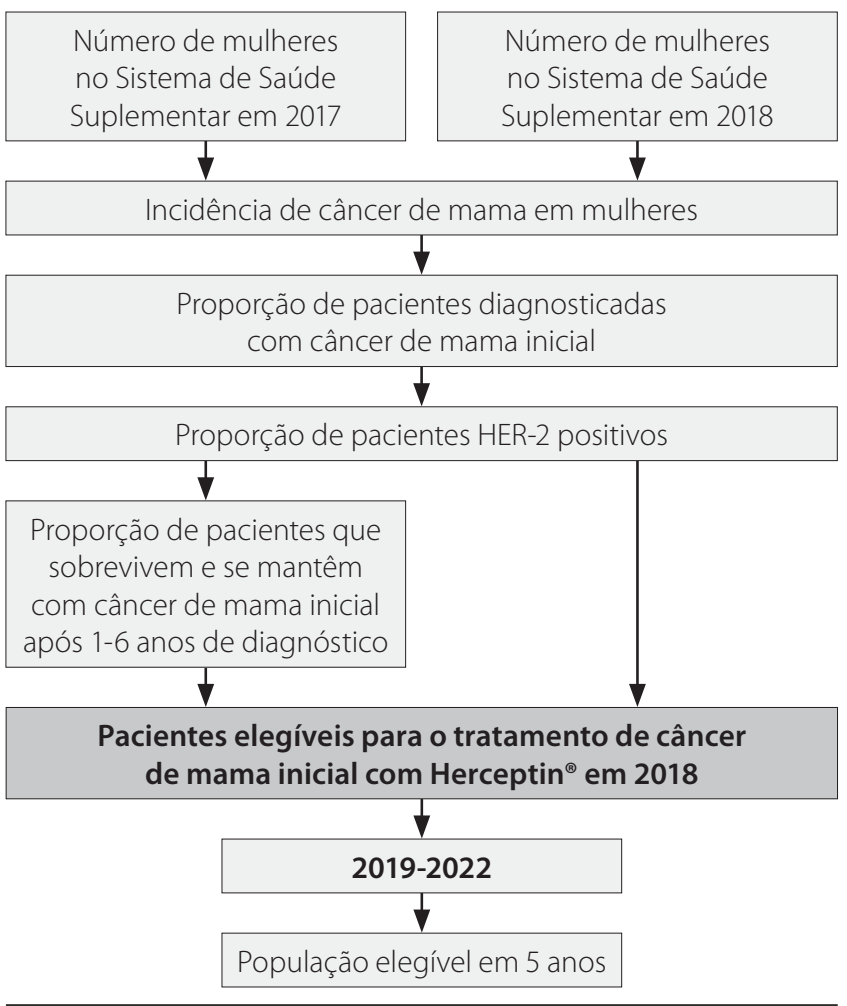

Figura 1. Fluxograma de pacientes para a obtenção da população elegível ao tratamento de câncer de mama inicial com trastuzumabe.

mento foram $5 \%, 10 \%, 0 \%, 7 \%, 6 \%$ e $4 \%$ para um, dois, três, quatro, cinco e seis anos entre o diagnóstico até o início da doença, respectivamente (Perez et al., 2014).

O método utilizado para a estimativa da população elegível ao tratamento com trastuzumabe em primeira e segunda linha de doença metastática está sumarizado na Figura 2.

\section{Tratamentos}

Considerou-se que os pacientes com câncer de mama inicial receberam trastuzumabe em associação com docetaxel ou paclitaxel. De acordo com os regimes utilizados no estudo HANNAH e as orientações de bula, o trastuzumabe é administrado em conjunto com a quimioterapia no primeiro dia de um ciclo de 21 dias, durante quatro ciclos. Após o tratamento neoadjuvante ou adjuvante em combinação com quimioterapia, o trastuzumabe é administrado em monoterapia até que complete um ano de tratamento (F. Hoffmann-La Roche Ltd, 2018a; Ismael et al., 2012). Assumiu-se que 50\% dos pacientes recebiam docetaxel e $50 \%$ recebiam paclitaxel.

Em todos os regimes, as doses de trastuzumabe IV foram de $8 \mathrm{mg} / \mathrm{kg}$ no primeiro ciclo e de $6 \mathrm{mg} / \mathrm{kg}$ nos ciclos subsequentes. Para o trastuzumabe SC, foram consideradas doses fixas de $600 \mathrm{mg}$, conforme recomendação de bula ( $F$. Hoffmann-La Roche Ltd, 2018a). A dose de docetaxel foi de $100 \mathrm{mg} / \mathrm{m}^{2}$ e a de paclitaxel, de $175 \mathrm{mg} / \mathrm{m}^{2}$. 


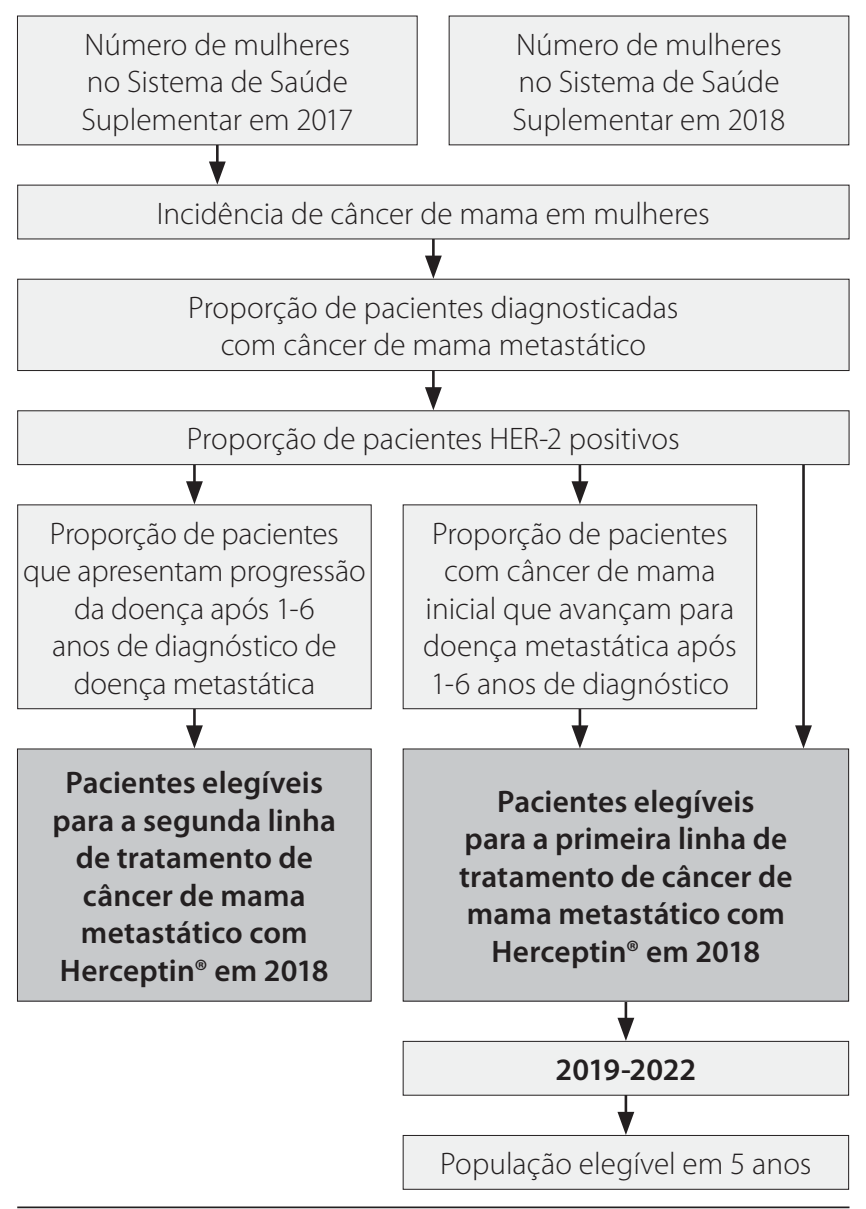

Figura 2. Fluxograma de pacientes para a obtenção da população elegível ao tratamento de câncer de mama metastático com trastuzumabe.

Para o cálculo das doses, utilizaram-se as médias de superfície corpórea $\left(1,67 \mathrm{~m}^{2}\right)$ e peso $(63,8 \mathrm{~kg})$ das mulheres adultas no Brasil, divulgados pelo IBGE (IBGE, 2011).

Para o câncer de mama metastático, em primeira linha de tratamento, considerou-se que os pacientes receberam trastuzumabe em associação com docetaxel ou paclitaxel.

Esses pacientes receberam seis ciclos de quimioterapia e então passaram a receber trastuzumabe em monoterapia até a progressão da doença. O tempo mediano até a progressão da doença foi de 6,9 meses para os pacientes que receberam trastuzumabe mais paclitaxel e de 11,7 meses para os que receberam a associação com docetaxel (Slamon et al., 2001; Marty et al., 2005).

Em segunda linha, os pacientes receberam trastuzumabe em monoterapia em ciclos de 21 dias durante 11 meses (o tempo mediano até a progressão da doença) (Cobleigh et al., 1999).

\section{Cenários e perspectivas}

Foram criados dois cenários para o câncer de mama inicial e para o metastático: cenário $A$, em que apenas o trastuzumabe IV é utilizado, e cenário $B$, com incorporação progressi- va de trastuzumabe SC. Foram estimados os custos médicos diretos para cada um dos cenários e o resultado foi apresentado como a diferença de custo entre os dois cenários.

Foram adotadas quatro perspectivas dentro do Sistema de Saúde Suplementar brasileiro: 1) Sistema de Saúde Suplementar como um todo; 2) Operadora de Saúde de Grande Porte ( $\geq 100$ mil beneficiários); 3) Operadora de Saúde de Médio Porte (20 mil a 100 mil beneficiários); 4) Operadora de Saúde de Pequeno Porte ( $<20$ mil beneficiários). O porte das operadoras de saúde foi definido com base no número de beneficiários, conforme estabelecido pela ANS.

Foram considerados os recursos e custos médicos diretos relacionados ao tratamento, incluindo: custo dos medicamentos oncológicos, materiais utilizados para administração, medicamentos de suporte e taxas administrativas e de honorários médicos.

Os materiais utilizados para administração de cada um dos regimes e suas quantidades foram estimados por uma enfermeira membro da equipe de uma clínica de oncologia do Sistema De Saúde Suplementar e validados por uma segunda equipe de outra clínica e por dois médicos oncologistas. Assumiu-se que $50 \%$ dos pacientes fazem administração via port-a-cath e 50\%, via acesso venoso periférico.

Os custos dos materiais foram extraídos da tabela Brasíndice e os custos dos medicamentos foram obtidos por meio da lista de preços publicada pela Câmara de Regulação do Mercado de Medicamentos (CMED), publicada em 10 de agosto de 2018, com a alíquota de 18\% para ICMS. O preço utilizado foi calculado a partir da média de todas as marcas disponíveis.

A taxa de sala e os honorários médicos relativos à administração de terapia imuno-oncológica IV e SC foram obtidos por meio de uma pesquisa realizada com operadoras de planos de saúde. Para isso, foi realizada uma pesquisa com 28 operadoras de planos de saúde de todo o Brasil, contatadas via e-mail e/ou telefone. A partir dos resultados, determinaram-se quais parâmetros seriam utilizados para a construção desse modelo de impacto orçamentário. Para medicamentos intravenosos e subcutâneos, utilizou-se o valor mediano do mercado.

Para o reembolso de medicamentos, a maioria das operadoras de planos de saúde (40\%) relatou utilizar a lista CMED. Para materiais, 60\% utilizam SIMPRO ou Brasíndice. O custo mediano para administração IV foi de R\$ R\$ 103,50 (valor mínimo: $R \$$ 0; valor máximo: $R \$ 645,00$ ) e o custo para administração SC de R\$ 9,00 (variação de R\$ 0 - a R\$ 307,70). Os custos de honorários médicos para a administração IV e SC foram de $R \$ 154,01$ (variação de $R \$ 50,00$ - a $R \$ 673,68$ ) e $R \$ 0,00$ (variação de R\$ 0 - a R\$ 165,94), respectivamente.

Para a estimativa do impacto orçamentário em cada uma das perspectivas, adotou-se um horizonte temporal de cinco anos. Os resultados foram reportados anualmente e também de maneira cumulativa ao final do período. 
No market-share, foi considerada a incorporação progressiva de trastuzumabe SC, com 20\% no primeiro ano, 40\% no segundo, $60 \%$ no terceiro, $80 \%$ no quarto e $100 \%$ no quinto.

\section{Análise de sensibilidade}

Foi realizada uma análise de sensibilidade determinística univariada para avaliar a influência da incerteza e variabilidade dos parâmetros utilizados no modelo nos resultados.

\section{Resultados}

\section{Estimativa de pacientes}

O número total de beneficiários e o número de mulheres por porte de operadora são apresentados na Tabela 1.

Os números estimados de pacientes elegíveis para os tratamentos são, no total, 31.589 pacientes na Saúde Suplementar, 284 em uma operadora de grande porte, 30 em uma de médio porte e 6 em uma de pequeno porte. Os resultados de pacientes elegíveis para o tratamento de câncer de mama inicial e metastático na primeira e segunda linha estão descritos na Tabela 2.

\section{Economia de recursos}

Os custos totais, que englobam o custo do medicamento, medicamentos de suporte, materiais, taxas e honorários, uti-

Tabela 1. Número de beneficiários por porte de operadora de saúde

\begin{tabular}{lcc}
\hline $\begin{array}{l}\text { Porte da operadora } \\
\text { de saúde }\end{array}$ & $\begin{array}{l}\text { Número médio de } \\
\text { beneficiários (DP) }\end{array}$ & Número de mulheres \\
\hline Grande porte & $431.656(688.177)$ & 228.778 \\
\hline Médio porte & $45.100(21.218)$ & 23.903 \\
\hline Pequeno porte & $7.589(5.887)$ & 4.022 \\
\hline
\end{tabular}

DP: desvio-padrão.

Tabela 2. Resultado do número de pacientes elegíveis em cada operadora e no Sistema de Saúde Suplementar

\begin{tabular}{lcccc}
\hline & $\begin{array}{c}\text { Saúde } \\
\text { Suplementar }\end{array}$ & $\begin{array}{c}\text { OPS Porte } \\
\text { Grande }\end{array}$ & $\begin{array}{c}\text { Médio } \\
\text { Mérte }\end{array}$ & $\begin{array}{c}\text { OPS Porte } \\
\text { Pequeno }\end{array}$ \\
\hline $\begin{array}{l}\text { Pacientes com } \\
\text { doença inicial }\end{array}$ & 28.027 & 253 & 26 & 5 \\
\hline $\begin{array}{l}\text { Pacientes } \\
\text { com doença } \\
\text { metastática 1L }\end{array}$ & 3.376 & 30 & 4 & 1 \\
\hline $\begin{array}{l}\text { Pacientes } \\
\text { com doença } \\
\text { metastática 2L }\end{array}$ & 186 & 2 & 0 & 0 \\
\hline $\begin{array}{l}\text { Total de } \\
\text { Pacientes }\end{array}$ & $\mathbf{3 1 . 5 8 9}$ & $\mathbf{2 8 4}$ & $\mathbf{3 0}$ & $\mathbf{6}$ \\
\hline
\end{tabular}

1L: primeira linha de tratamento; $2 \mathrm{~L}$ : segunda linha de tratamento.

OPS: Operadoras de Planos de Saúde. lizados para o cálculo do impacto orçamentário referente aos regimes do tratamento IV e SC, estão descritos na Tabela 3.

A análise de impacto orçamentário mostra potencial economia de recursos no Sistema de Saúde Suplementar. Ao longo de cinco anos, com a incorporação progressiva de trastuzumabe SC para o tratamento de pacientes com câncer de mama, estima-se uma economia total de recursos de $R \$ 962.725 .065,38$, R\$ 14.558.810,73, R\$1.527.774,61 e R\$255.833,12 para o Sistema de Saúde Suplementar, operadora de saúde de grande, uma de médio e uma de pequeno porte, respectivamente (Figura 3).

A Tabela 4 mostra o impacto orçamentário em cada uma das indicações - câncer de mama inicial e metastático HER-2 positivo. A substituição da administração do trastuzumabe IV pela forma SC no tratamento do câncer de mama inicial HER-2 positivo pode trazer economia de até $\mathbf{R}$ 929.572.032,75 e de até $R \$ 33.153 .032,63$ no câncer de mama metastático para o Sistema de Saúde Suplementar em cinco anos.

\section{Análise de sensibilidade}

Em todas as perspectivas, os parâmetros cuja variação mais influenciou os resultados foram a incidência de câncer de mama, o tempo de tratamento em monoterapia para câncer de mama inicial e as taxas de sala (Figura 4).

\section{Discussão}

Os resultados da análise de impacto orçamentário demonstraram que trastuzumabe SC tem potencial de reduzir os custos de tratamento do câncer de mama inicial e metastático no Sistema de Saúde Suplementar do Brasil.

A terapia com trastuzumabe SC apresentou menor custo se comparado ao trastuzumabe IV no tratamento de câncer de mama inicial e metastático no Sistema de Saúde Suple-

Tabela 3. Custo total por regime de tratamento

\begin{tabular}{|c|c|c|c|}
\hline \multirow{3}{*}{ Trastuzumabe monoterapia } & \multirow{2}{*}{ IV } & PTC & $R \$ 13.017,74$ \\
\hline & & AVP & $R \$ 12.579,67$ \\
\hline & SC & - & $\mathrm{R} \$ 10.132,54$ \\
\hline \multirow{4}{*}{ Trastuzumabe + Docetaxel } & \multirow{2}{*}{ PTC } & IV & $\mathrm{R} \$ 20.189,11$ \\
\hline & & SC & $R \$ 17.976,70$ \\
\hline & \multirow{2}{*}{ AVP } & IV & $R \$ 19.734,69$ \\
\hline & & SC & $R \$ 17.523,36$ \\
\hline \multirow{4}{*}{ Trastuzumabe + Paclitaxel } & \multirow{2}{*}{ PTC } & IV & $R \$ 18.697,92$ \\
\hline & & SC & $R \$ 16.583,12$ \\
\hline & \multirow{2}{*}{ AVP } & IV & $\mathrm{R} \$ 18.232,27$ \\
\hline & & SC & $\mathrm{R} \$ 16.135,12$ \\
\hline
\end{tabular}

PTC: administração via port-a-cath; AVP: administração via acesso venoso periférico; IV: intravenoso; SC: subcutâneo. Considerando dose de trastuzumabe de $6 \mathrm{mg} / \mathrm{kg}$ 


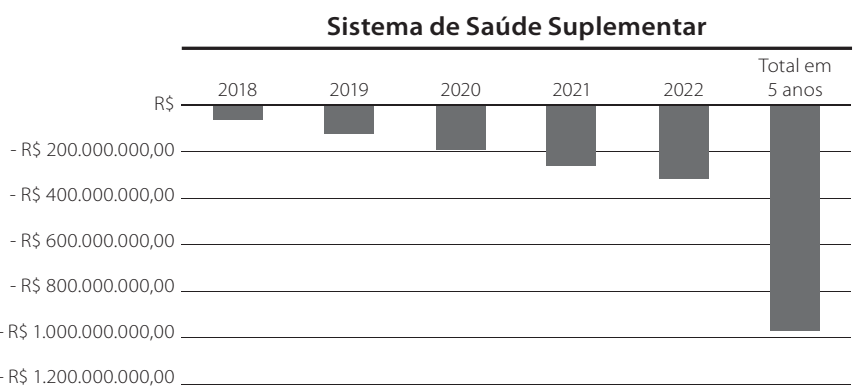

Operadora de grande porte

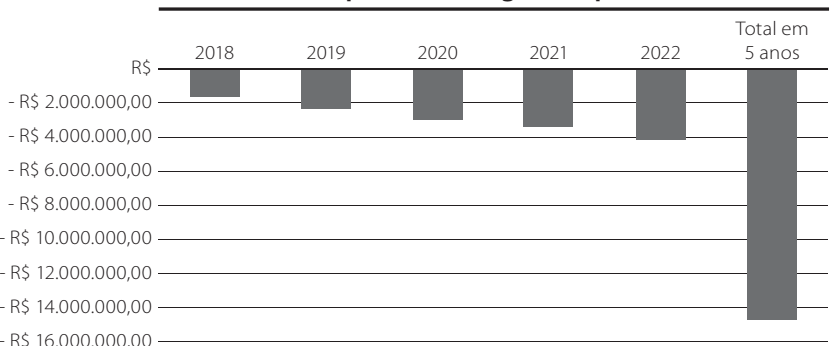

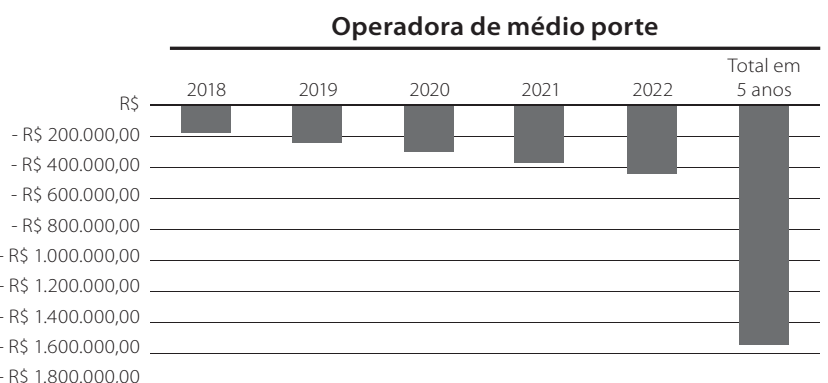

Operadora de pequeno porte

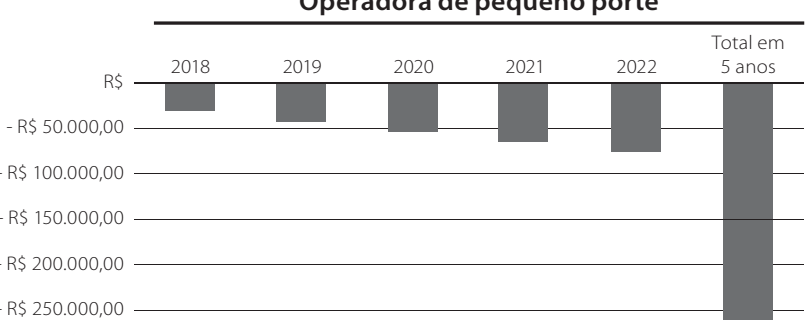

R\$ $300.000,00$

Figura 3. Impacto orçamentário da incorporação progressiva de trastuzumabe no Sistema de Saúde Suplementar e em operadoras de planos de saúde de grande, médio e pequeno porte.

Tabela 4. Resultado do impacto orçamentário do tratamento do câncer de mama com trastuzumabe SC

\begin{tabular}{lccc}
\hline & Custo do IV & Custo do SC & Impacto Orçamentário \\
\hline Câncer de mama inicial & & & $-R \$ 929.572 .032,75$ \\
\hline SSS & $R \$ 7.401 .723 .688,30$ & $R \$ 6.472 .151 .655,55$ & $-R \$ 8.393 .207,66$ \\
\hline Grande Porte & $R \$ 66.830 .973,55$ & $R \$ 58.437 .765,90$ & $-R \$ 876.933,64$ \\
\hline Médio Porte & $R \$ 6.982 .590,09$ & $R \$ 6.105 .656,45$ & $-R \$ 147.562,07$ \\
\hline Pequeno Porte & $R \$ 1.174 .964,00$ & $R \$ 1.027 .401,93$ & $-R \$ 33.153 .032,63$ \\
\hline Câncer de mama metastático & & & $-R \$ 6.165 .603,08$ \\
\hline SSS & $R \$ 687.415 .703,20$ & $R \$ 654.262 .670,58$ & $-R \$ 650.840,97$ \\
\hline Grande Porte & $R \$ 11.926 .889,84$ & $R \$ 5.761 .286,76$ & $-R \$ 108.271,05$ \\
\hline Médio Porte & $R \$ 1.259 .373,51$ & $R \$ 608.532,53$ & $R \$ 101.127,05$ \\
\hline Pequeno Porte & $R \$ 209.398,10$ & & \\
\hline
\end{tabular}

SSS: Sistema de Saúde Suplementar; grande, médio e pequeno porte das operadoras de saúde.

mentar em geral e também nas operadoras de pequeno, médio e grande porte individualmente. Entre os custos avaliados, a taxa de sala e honorários médicos foram alguns dos fatores que mais contribuíram para essa economia.

Foi demonstrada que a incorporação de trastuzumabe SC traz economia de $12,5 \%$ no custo do tratamento do câncer de mama inicial e de até 51,7\% no câncer de mama metastático HER-2 positivo. No total, a economia potencial para o Sistema de Saúde Suplementar é de até R\$ 962,7 milhões ao longo de cinco anos. Dado o cenário econômico atual e a necessidade de uso racional de recursos, esse resultado mostra uma importante estratégia a ser considerada para a redução do custo do tratamento de câncer de mama HER-2 positivo no país.
De fato, outros estudos vêm demonstrando que a substituição de medicamentos IV por SC reduz os custos relacionados à administração dos medicamentos. Olsen et al. estimaram o custo da administração SC versus IV de trastuzumabe e demonstraram que, na Dinamarca, a forma SC é 47,7\% mais econômica se comparada à IV em 17 ciclos de tratamento (Olsen et al., 2018). Tjalma et al. também avaliaram o custo de administração de trastuzumabe SC versus IV nas indicações de câncer de mama inicial e metastático e demonstraram, na Bélgica, que a forma SC pode trazer economia de \$231,73 por administração se comparada à IV, o que pode ser traduzido como redução total de $\$ 4.171,06$ em um tratamento com 18 ciclos (Tjalma et al., 2018). Além disso, a dosagem fixa da administração do trastuzumabe SC confere certeza à 

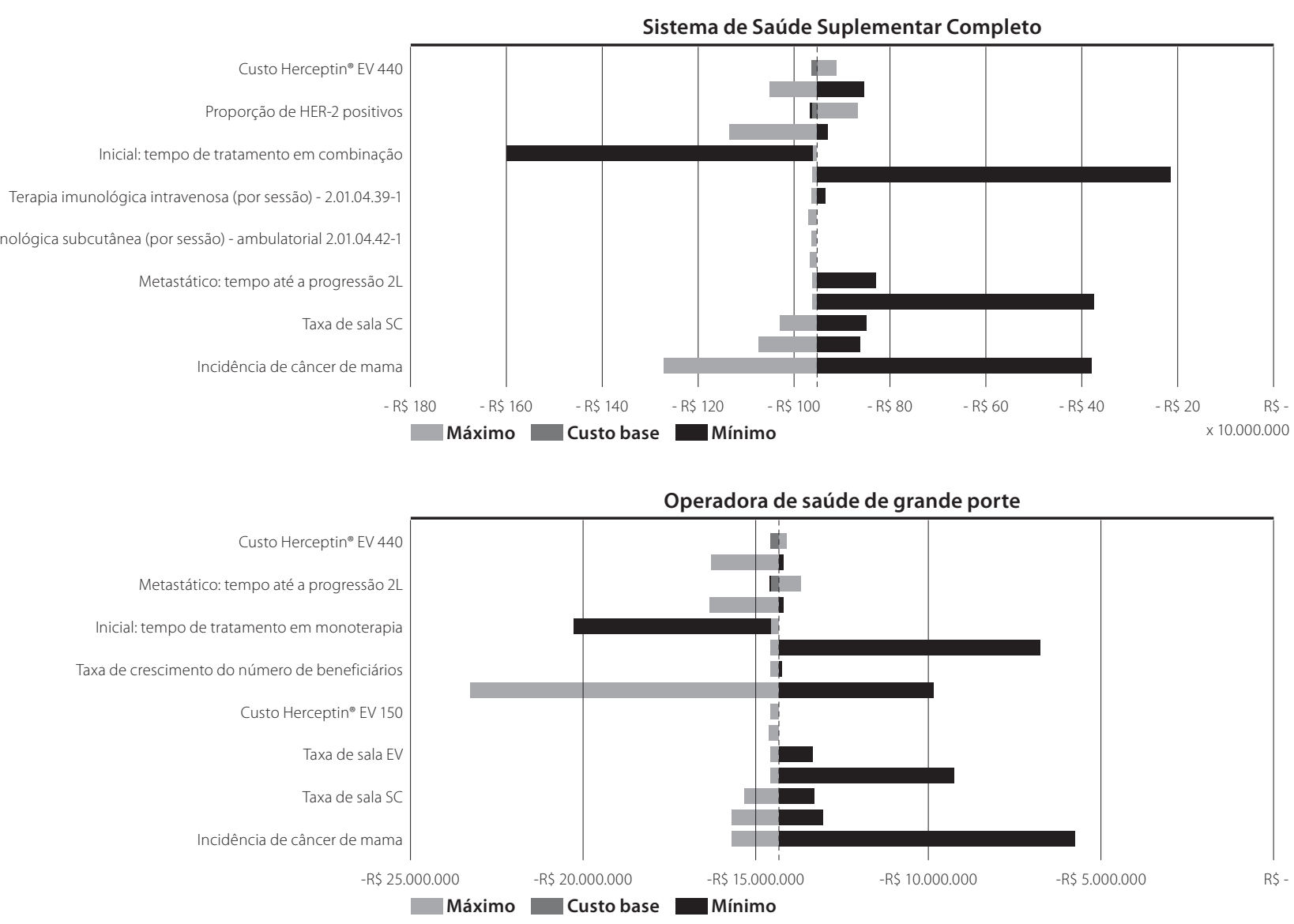

Terapia imunológica subcutânea (por sessão) - ambulatorial 2.01.04.42-1

Metastático: tempo até a progressão

Operadora de saúde de médio porte
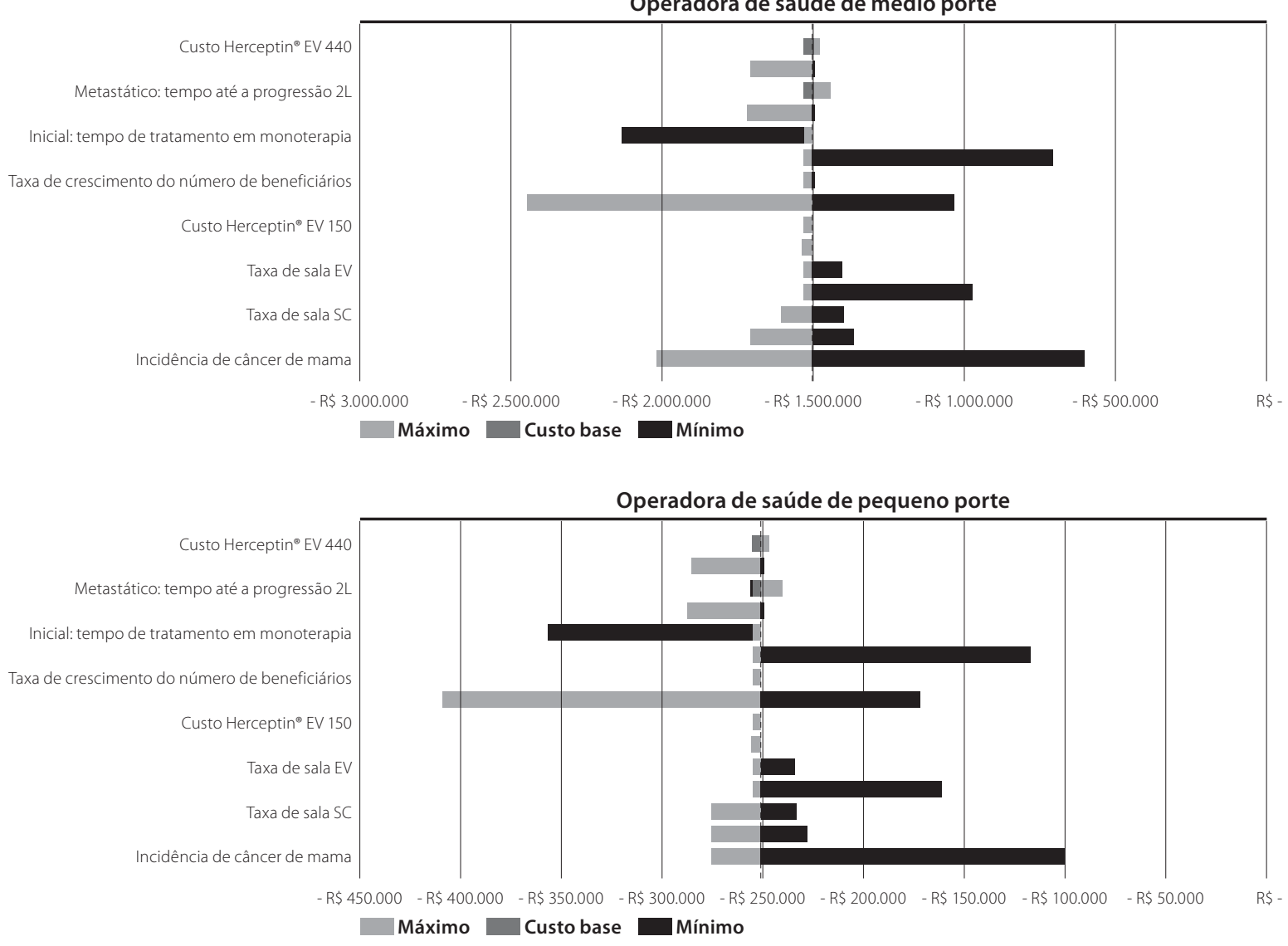

Figura 4. Análise de sensibilidade. 
estimativa do impacto orçamentário, já que a posologia independe do peso corpóreo do paciente, ao mesmo tempo que não causa desperdício da droga (Tjalma et al., 2018).

Cock et al. avaliaram o tempo médio para a infusão de trastuzumabe SC com a utilização de dispositivo injetável de uso único (DIU) ou seringa portátil (SP) versus trastuzumabe IV. O tempo economizado na cadeira de infusão pelos pacientes foi de 57 minutos com a administração SC DIU se comparado com a IV (variação entre os países avaliados de 47-86; $p<0,0001)$. A utilização da SP trouxe economia de 55 minutos $(40-81 ; \mathrm{p}<0,0001)$ se comparada à IV. O tempo gasto pelos profissionais de saúde para administração apresentou redução média de 13 minutos por sessão com a aplicação SC DIU (variação entre os países avaliados de 4-16; $p<0,0001)$ e de 17 minutos com a forma SC SP $(5-28 ; p<$ $0,0001)$ versus IV. Portanto, houve economia do tempo que o paciente passa na cadeira de infusão e do tempo que os profissionais de saúde gastam para a aplicação de trastuzumabe SC se comparado à forma IV para aplicação com DIU ou SP (De Cock et al., 2016).

Ademais, Olsen et al. avaliaram o tempo de administração SC versus IV e verificaram que foi consideravelmente menor (25 minutos versus 85 minutos, respectivamente) (Olsen et al., 2018). Wiebren et al. estimaram que o tempo total de preparação e administração de trastuzumabe IV foi 4,07 vezes maior que a SC. O tempo que um paciente passa no hospital foi $71 \%$ menor com a forma SC. O tempo médio total economizado por profissionais da saúde para administração SC foi de 53,7 minutos e de 122,5 minutos para pacientes (Tjalma et al., 2018). Logo, a forma SC também traz considerável economia de tempo na preparação e infusão da droga, o que beneficia tanto pacientes quanto profissionais de saúde.

Destaca-se ainda a preferência dos pacientes e profissionais da saúde pela administração SC. No câncer de mama inicial, a administração de trastuzumabe SC foi a preferência de 88,9\% (415/467) pacientes [IC 95\%; 85,7-91,6; $\mathrm{p}<0,0001$; teste bicaudal contra a hipótese nula de preferência do SC de 65\%] versus 9,6\% (45/467) IV [IC 95\%; 7-13]; 1,5\% (7/467) indicou não ter preferência pela via de administração [IC 95\%; 1-3]. Essa preferência também é superior entre os profissionais da saúde; 77\% (181/235) responderam que sentem maior satisfação com a via SC [IC 95\%;71,1-82,2] versus 3\% (7/235) com IV [IC 95\%; 1,2-6,0]. Os demais 20\% (47/235) indicaram não ter alguma preferência [IC 95\%; 15,1-25,7] (Pivot et al., 2014). Pivot et al. fizeram essa análise com pacientes com câncer de mama metastático e concluíram que a via SC é preferível por 85,9\% (79/92) pacientes (IC 95\%; 78,8-93,0 p < 0,0001) versus 14,1\% (13/92) IV [IC 95\%; 7,0-21,3]. Entre os profissionais da saúde a preferência pelo SC, foi de 63,6\% (56/88) [IC 95\%; 53,6-73,7]. Portanto, a administração SC é a preferível por pacientes e profissionais da saúde tanto no tratamento do câncer de mama inicial quanto metastático (Pivot et al., 2017).
Algumas limitações do estudo podem ser citadas, como a incerteza da incidência de câncer de mama no país, principalmente pela dificuldade em se ter dados de epidemiologia provenientes da Saúde Suplementar. Além disso, as taxas de sala e os preços de medicamentos e materiais pagos pelas operadoras de planos de saúde diferem muito entre si. A pesquisa com as operadoras de saúde forneceu dados reais do setor, porém há a incerteza associada à acurácia das pessoas ao responderem à pesquisa e ao tamanho da amostra (uma vez que o setor possui 756 operadoras de planos de saúde). Entretanto, mesmo com máximo custo de sala reportado, há economia de recursos em todos os cenários (sistema de saúde suplementar como um todo e operadoras de grande, médio e pequeno porte), conferindo credibilidade e robustez aos resultados apresentados.

\section{Conclusão}

Trastuzumabe SC no tratamento de câncer de mama inicial e metastático HER-2 positivo tem potencial de gerar economia de recursos, comparado ao trastuzumabe IV no sistema de saúde suplementar do Brasil, sendo a taxa de sala e honorários médicos os principais motivos dessa economia.

\section{Referências bibliográficas}

ANS - Agência Nacional de Saúde Suplementar. Benef. Assistência Médica segundo Operadora. 2017a. Disponível em: http://www.ans.gov.br/ anstabnet/cgi-bin/tabnet?dados/tabnet_cc.def. Acesso em: 30 jan. 2019.

ANS - Agência Nacional de Saúde Suplementar. Beneficiários de planos suplementares de saúde, por cobertura assistencial (Brasil - 2007-2017). 2017b. Disponível em: http://www.ans.gov.br/perfil-do-setor/dadosgerais. Acesso em: 30 jan. 2019.

ANS - Agência Nacional de Saúde Suplementar. Taxa de crescimento (\%) do número de beneficiários em relação a dezembro do ano anterior (Brasil 2007-2017) 2017c. Disponível em: http://www.ans.gov.br/perfil-do-setor/ dados-gerais. Acesso em: 30 jan. 2019.

Arias VEA, Gobbi H, loshii SO, Scapulatempo C, Paz ARD, Silva VDD, et al. Assessment of HER-2 status in invasive breast cancer in Brazil. Rev Assoc Med Bras (1992). 2017;63(7):566-74.

Brasil. Ministério da Saúde. Secretaria de Ciência, Tecnologia e Insumos Estratégicos. Departamento de Ciência e Tecnologia. Diretrizes metodológicas: análise de impacto orçamentário: manual para o Sistema de Saúde do Brasil. 1a ed. Brasília; 2014.

Cameron D, Piccart-Gebhart MJ, Gelber RD, Procter M, Goldhirsch A, de Azambuja E, et al. 11 years' follow-up of trastuzumab after adjuvant chemotherapy in HER2-positive early breast cancer: final analysis of the HERceptin Adjuvant (HERA) trial. Lancet. 2017;389(10075):1195-205.

Cobleigh MA, Vogel CL, Tripathy D, Robert NJ, Scholl S, Fehrenbacher L, et al., Multinational study of the efficacy and safety of humanized antiHER2 monoclonal antibody in women who have HER2-overexpressing metastatic breast cancer that has progressed after chemotherapy for metastatic disease. J Clin Oncol. 1999;17(9):2639-48.

De Cock E, Pivot X, Hauser N, Verma S, Kritikou P, Millar D, et al. A time and motion study of subcutaneous versus intravenous trastuzumab in patients with HER2-positive early breast cancer. Cancer Med. 2016;5(3):389-97. 
F. Hoffmann-La Roche Ltd. Bula do Medicamento - Herceptin SC. 2018a. Disponível em: http://www.anvisa.gov.br/datavisa/fila_bula/ frmVisualizarBula.asp?pNuTransacao=113342018\&pldAnexo=10415252. Acesso em: 30 jan. 2019.

F. Hoffmann-La Roche Ltd. Bula do Medicamento - Pertuzumabe. 2018b. Disponível em: http://www.anvisa.gov.br/datavisa/fila_bula/ frmVisualizarBula.asp?pNuTransacao=8559662018\&pldAnexo=10750914. Acesso em: 30 jan. 2019.

Hudis CA. Trastuzumab - mechanism of action and use in clinical practice. N Engl J Med. 2007;357(1):39-51.

IBGE - Instituto Brasileiro de Geografia Estatística. Pesquisa de orçamentos familiares 2008-2009: análise do consumo alimentar pessoal no Brasil. IBGE, Coordenação de Trabalho e Rendimento. 2011.

Inca - Instituto Nacional do Câncer José Alencar Gomes da Silva. Estimativa 2018: incidência de câncer no Brasil. Rio de Janeiro; 2017.

Ismael G, Hegg R, Muehlbauer S, Heinzmann D, Lum B, Kim SB, et al. Subcutaneous versus intravenous administration of (neo)adjuvant trastuzumab in patients with HER2-positive, clinical stage I-III breast cancer (HannaH study): a phase 3, open-label, multicentre, randomised trial. Lancet Oncol. 2012;13(9):869-78.

Jackisch C, Hegg R, Stroyakovskiy D, Ahn JS, Melichar B, Chen SC, et al. HannaH phase III randomised study: Association of total pathological complete response with event-free survival in HER2-positive early breast cancer treated with neoadjuvant-adjuvant trastuzumab after 2 years of treatment-free follow-up. Eur J Cancer. 2016;62:62-75.

Liedke PE, Finkelstein DM, Szymonifka J, Barrios CH, Chavarri-Guerra Y, Bines J, et al. Outcomes of breast cancer in Brazil related to health care coverage: a retrospective cohort study. Cancer Epidemiol Biomarkers Prev. 2014;23(1):126-33.

Marty M, Cognetti F, Maraninchi D, Snyder R, Mauriac L, Tubiana-Hulin M, et al. Randomized phase II trial of the efficacy and safety of trastuzumab combined with docetaxel in patients with human epidermal growth factor receptor 2-positive metastatic breast cancer administered as firstline treatment: the M77001 study group. J Clin Oncol. 2005;23(19):4265-74.

Mitri Z, Constantine T, O'Regan R. The HER2 Receptor in Breast Cancer: Pathophysiology, Clinical Use, and New Advances in Therapy. Chemother Res Pract. 2012;2012:743193.
Olsen J, Jensen KF, Olesen DS, Knoop A. Costs of subcutaneous and intravenous administration of trastuzumab for patients with HER2positive breast cancer. J Comp Eff Res. 2018;7(5):411-9.

Perez EA, Romond EH, Suman VJ, Jeong JH, Sledge G, Geyer CE Jr, et al. Trastuzumab plus adjuvant chemotherapy for human epidermal growth factor receptor 2-positive breast cancer: planned joint analysis of overall survival from NSABP B-31 and NCCTG N9831. J Clin Oncol. 2014;32(33):3744-52.

Pivot X, Gligorov J, Müller V, Curigliano G, Knoop A, Verma S, et al. Patients' preferences for subcutaneous trastuzumab versus conventional intravenous infusion for the adjuvant treatment of HER2-positive early breast cancer: final analysis of 488 patients in the international, randomized, two-cohort PrefHer study. Ann Oncol. 2014;25(10):1979-87.

Pivot X, Spano JP, Espie M, Cottu P, Jouannaud C, Pottier V, et al. Patients' preference of trastuzumab administration (subcutaneous versus intravenous) in HER2-positive metastatic breast cancer: Results of the randomised MetaspHer study. Eur J Cancer. 2017;82:230-6.

Slamon DJ, Leyland-Jones B, Shak S, Fuchs H, Paton V, Bajamonde A, et al. Use of chemotherapy plus a monoclonal antibody against HER2 for metastatic breast cancer that overexpresses HER2. N Engl J Med. 2001;344(11):783-92.

Sullivan SD, Mauskopf JA, Augustovski F, Jaime Caro J, Lee KM, Minchin M, et al. Budget impact analysis-principles of good practice: report of the ISPOR 2012 Budget Impact Analysis Good Practice II Task Force. Value Health. 2014;17(1):5-14.

Tjalma WAA, Van den Mooter T, Mertens T, Bastiaens V, Huizing MT, Papadimitriou K. Subcutaneous trastuzumab (Herceptin) versus intravenous trastuzumab for the treatment of patients with HER2positive breast cancer: A time, motion and cost assessment study in a lean operating day care oncology unit. Eur J Obstet Gynecol Reprod Biol. 2018;221:46-51.

WHO - World Health Organization. Globocan 2012: Estimated cancer incidence, mortality and prevalence worldwide in 2012 [Internet]. Incidence/Mortality > rates: populations by cancer. 2012. Disponível em: http://globocan.iarc.fr/Pages/summary_table_site_sel.aspx. Acesso em: 30 jan. 2019 